\title{
Conjunctival Fungal Ball Mimicking Melanoma
}

\author{
Katherine Chuang, $\mathrm{MD}^{1}$, Alexander Vortmeyer, MD ${ }^{2}$, Shaohui Liu, MD, PhD ${ }^{1}$
}

1 Glick Eye Institute, Indiana University School of Medicine, Indianapolis, Indiana

2 Clinical Pathology \& Laboratory Medicine, Indiana University School of Medicine, Indianapolis, Indiana

A 74-year-old woman was referred with suspicion of conjunctival melanoma because 2 pigmented lesions were noted in the superior conjunctiva for several months. During examination with eyelid eversion to look for other satellite lesions, the patient squeezed forcefully. The larger lesion became mobile and moved across the cornea to the inferior conjunctiva (Fig A, B, blue arrows show indented conjunctiva at the original location; yellow arrows show the mobile lesion). This larger lesion was removed from the eye with forceps, placed on a piece of filter paper and sent to pathology. Periodic acid-Schiff staining revealed a conglomerate of fungal organisms (Fig C). The smaller lesion, which was partially embedded under the conjunctiva, was then completely removed under a slit lamp with sterile forceps (Fig D). Culture of the smaller lesion grew Cladosporium species. Because both lesions were completely removed, antifungal therapy was not initiated. There was no recurrence. She was immunocompetent and denied any eye injury history. (Magnified version of Fig A-D is available online at www.aaojournal.org).

This is the authors' manuscript of the work published in final form as:

Chuang, K., Vortmeyer, A., \& Liu, S. (2020). Conjunctival Fungal Ball Mimicking Melanoma. Ophthalmology, 127(4), 534. https://doi.org/10.1016/j.ophtha.2019.12.027 

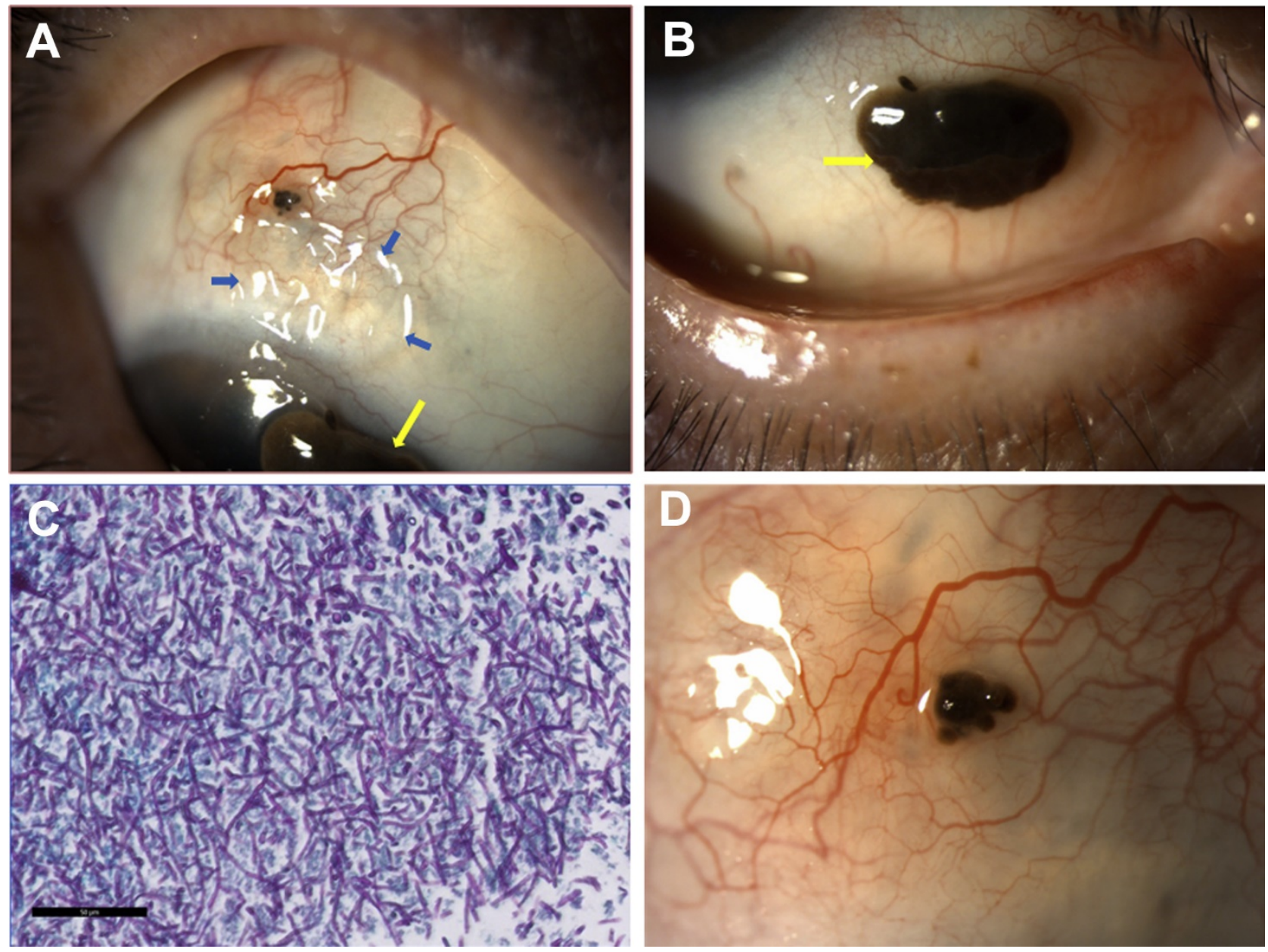